\title{
Nonequilibrium temperature measurement in a thermal conduction process
}

\author{
Puneet Kumar Patra* and Romesh C. Batra ${ }^{\dagger}$ \\ Department of Biomedical Engineering and Mechanics, M/C 0219, \\ Virginia Polytechnic Institute and State University, Blacksburg, Virginia 24061, USA
}

(Received 16 July 2016; published 10 January 2017)

\begin{abstract}
We identify the temperature being measured by a thermometer in a nonequilibrium scenario by studying heat conduction in a three-dimensional Lennard-Jones (LJ) system whose two ends are kept at different temperatures. It is accomplished by modeling the thermometer particles also with the LJ potential but with added tethers to prevent their rigid body motion. These models of the system and the thermometer mimic a real scenario in which a mechanical thermometer is "inserted" into a system and kept there long enough for the temperature to reach a steady value. The system is divided into five strips, and for each strip the temperature is measured using an embedded thermometer. Unlike previous works, these thermometers are small enough not to alter the steady state of the nonequilibrium system. After showing initial transients, the thermometers eventually show steady-state conditions with the subregions of the system and provide values of the different temperature definitions-kinetic, configurational, dynamical, and higher-order configurational. It is found that their kinetic and the configurational temperatures are close to the system's kinetic temperature except in the two thermostatted regions. In the thermostatted regions, where the system's kinetic and the configurational temperatures are significantly different, the thermometers register a temperature substantially different from either of these two values. With a decrease in the system density and size, these differences between the kinetic and the configurational temperatures of the thermometer become more pronounced.
\end{abstract}

DOI: 10.1103/PhysRevE.95.013302

\section{INTRODUCTION}

A challenge in nonequilibrium thermodynamics is extending the meaning of equilibrium variables to nonequilibrium scenarios [1-3]. Temperature is one such variable of interest [4]. Defining temperature and its measurement using thermometers relies upon the validity of the Zeroth Law of thermodynamics [5] - the mutual thermal equilibrium of three contacting bodies. It has been argued that this fundamental principle may not be applicable to away-from-equilibrium conditions [6,7]. To further complicate matters, almost every process of interest falls under the domain of nonequilibrium thermodynamics. Therefore, in this work, we ask the following question: What variable does a thermometer measure in a nonequilibrium process?

A nonequilibrium process may be either near-equilibrium or far-from-equilibrium. For the former, it is usually assumed that the local thermodynamic equilibrium (LTE) conditions prevail, and consequently the concepts of equilibrium thermodynamics, including variables such as temperature, are applicable locally [8-10]. However, thermometry even under LTE conditions is not straightforward, as we will see in the next section. Away from the local thermodynamic equilibrium regime, thermometry is even more daunting as one faces the problem of defining temperature. Consider, for example, the thermodynamic temperature $(T)$ [11] that is expressed in terms of the internal energy $(E)$ and the entropy $(S)$ by

$$
T=\left(\frac{\partial E}{\partial S}\right)_{V},
$$

where the partial derivative is performed at constant volume $V$. The entropy itself is undefined in a nonequilibrium process

\footnotetext{
*pkpatra@vt.edu

†rbatra@vt.edu
}

because of the multifractal nature of the phase space and the consequent divergence of $\log (f), f$ being the probability distribution function $[7,12,13]$.

Apart from the thermodynamic definition, temperature may be expressed in several different ways, with each definition having its own sets of problems in nonequilibrium settings. In molecular dynamics (MD), temperature has been synonymously associated with the kinetic temperature [11], $T_{K}$, defined (in a three-dimensional system) as

$$
\frac{3}{2} N k_{B} T_{K}=\sum_{i=1}^{3 N} \frac{1}{2} m v_{i}^{2},
$$

where $k_{B}$ is the Boltzmann constant, $N$ represents the total number of particles, $m$ is the mass of a particle, and $v_{i}$ is the velocity of the $i$ th particle. $T_{K}$ may also be viewed as the standard deviation of the normally distributed velocity distribution, making it possible to define higher-order kinetic temperatures [14]. The kinetic temperature is based on the instantaneous equilibration between the system and the thermometer particles [7] along with the equipartition theorem [5] - partitioning of the kinetic energy equally among the kinetic degrees of freedom. In other words, $T_{K}$ is synchronous with the ideal-gas thermometry. However, for a shock wave propagating in a medium [15], where the equipartition theorem is violated, $T_{K}$ shows directional dependence:

$$
T_{K}^{x x} \neq T_{K}^{y y} \neq T_{K}^{z z},
$$

$T_{K}^{i i}$ being the kinetic temperature along the $i$ th direction. The anisotropy in the kinetic temperature indicates that the Zeroth Law may not be valid in a far-from-equilibrium process. Nevertheless, a thermometer in such a scenario still registers a single reading. While some previous studies have argued for the equality between the ideal-gas thermometer and the local instantaneous mechanical kinetic temperature $[7,16]$, others 
have suggested that the kinetic temperature measured by a near-equilibrium thermometer differs from the average kinetic energy of the system $[17,18]$. Thus, again a question arises: Which variable is measured by a mechanical thermometer?

From a computational perspective, the kinetic temperature is both easy to calculate and has a local characteristic, unlike its configurational counterpart, $T_{C}$, which is expressed in terms of the configurational variables $[19,20]$ of the system:

$$
k_{B} T_{C}=\frac{\left\langle\nabla_{r} U \cdot \nabla_{r} U\right\rangle}{\left\langle\nabla_{r}^{2} U\right\rangle} .
$$

Here, the gradient operation on the potential energy, $U$, is performed with respect to coordinate $r$ of all particles, $\langle\cdots\rangle$ indicates average, and the dot in the numerator represents the scalar product between two vectors. $T_{C}$ is relevant for flowing nonequilibrium systems [20] as its evaluation does not depend upon the a priori knowledge of the streaming velocity. An accurate determination of $T_{K}$, on the other hand, requires the streaming velocity that makes its calculation more complicated [21,22]. The control of $T_{C}$ is also deemed to be useful for long-chain polymer molecules and proteins [20]. Unlike $T_{K}, T_{C}$ does not have a basis in the ideal-gas thermometry because (i) for perfect gases $T_{C}$ is undefined, and (ii) $T_{C}$ has a nonlocal characteristic, i.e., its value in a particular region is affected by its surroundings.

The definition of $T_{C}$ is a special case of a more general relationship [23]:

$$
k_{B} T_{D}=\frac{\langle\nabla E \cdot \nabla B\rangle}{\left\langle\nabla^{2} B\right\rangle},
$$

where $E$ equals the total energy of the system, and the gradient operation is performed with respect to both the coordinates and the momentum of the particles. Equation (5) defines the temperature, $T_{D}$, of the system in terms of an arbitrary scalar phase function $B$. Taking $B$ as the kinetic energy gives Eq. (2), while setting $B=U$ provides Eq. (4). Alternatively, if we set $B=E$, we get Rugh's temperature [24], $T_{R}$,

$$
k_{B} T_{R}=\frac{\langle\nabla E \cdot \nabla E\rangle}{\left\langle\nabla^{2} E\right\rangle} .
$$

The higher-order configurational temperatures [14] are defined by taking different variables for $B$ :

$$
B=U^{2} \Rightarrow k_{B} T_{C, 2}=\frac{\left\langle U \nabla_{r} U \cdot \nabla_{r} U\right\rangle}{\left\langle\nabla_{r} U \cdot \nabla_{r} U+U \nabla_{r}^{2} U\right\rangle} .
$$

The statistical-mechanical theory of thermometry based on $T_{K}$ and its derivatives relies on the instantaneous equilibration between the system and the thermometer due to the rapid elastic collisions between their constituents (kinetic theory of gases). On the other hand, thermometry based on $T_{C}$ and its derivatives does not allow equilibration through collisions, as system particles interacting through realistic potentials seldom collide with each other. The reason equilibrium thermometry provides a unique value of temperature is because in equilibrium all of the different expressions of temperature are equal [25]:

$$
T=T_{K}=T_{C}=T_{D}=T_{\text {higher order }} .
$$

However, there is no guarantee that it will be so in far-fromequilibrium processes, again raising a question on the variable being measured by the thermometer in nonequilibrium settings. The measurement of temperature also depends upon the nature of interaction between the system and a thermometer. Consider the case in which the thermometer and the system particles are highly repulsive vis-á-vis the case in which the particles are attractive. It is likely that the temperatures measured by the same thermometer in the two cases will be different.

In this article, we address (i) the merit of the statistical mechanical theory of thermometry, including the Zeroth Law, under LTE conditions by checking for the equality (and the cross equality) between the different temperature metrics of the system and the thermometer; (ii) whether this equality depends on the properties of the system (i.e., the system size, density, interaction, etc.); (iii) if the response at the boundaries is different from that in the system interior; and (iv) does a violation of LTE conditions (i.e., a disagreement between $T_{K}$ and $T_{C}$ ) have any bearing on the temperature of the thermometer. We explore these concepts through numerical simulations by considering the system and the thermometers to be composed of Lennard-Jones (LJ) particles. We focus on a near-equilibrium thermal conduction process engineered by two Nosé-Hoover thermostats. For both the system and the thermometer, we evaluate different measures of temperature, and we see which of them are in agreement with each other.

The rest of this paper is organized as follows. In the next section, we briefly review some of the previous attempts at answering the above-mentioned questions. Section III describes the simulation model and the methodology. After discussing the results in Sec. IV, conclusions are summarized in Sec. V.

\section{PREVIOUS EFFORTS IN DETERMINING NONEQUILIBRIUM TEMPERATURE}

Nonequilibrium molecular dynamics (NEMD) enables one to study the various aspects of nonequilibrium thermodynamics and to provide fundamental insights. Employing NEMD, Baranyai [17,18] explored nonequilibrium thermometry in two-dimensional systems. The thermometer consists of hundreds of firmly held fluid particles embedded in the system. The thermometer is free to translate, vibrate, and rotate within the system. The operational temperature, $T_{O}$, measured by the thermometer is taken to be its kinetic temperature. Baranyai tested this thermometer on problems related to shear flow and thermal conduction, and found that neither the system's kinetic temperature nor its configurational temperature equalled the operational temperature of the thermometer, possibly due to the thermometer size being a large fraction of the size of the system. Within the thermometer, thermal gradients and internal stresses exist [7], making it difficult to estimate the local temperature of a subregion of the system. Additionally, in a shear flow the local velocity changes with position, which makes a global operational temperature unrealistic. Thus it is not surprising to find that $T_{O}$ differs from both $T_{K}$ and $T_{C}$ of the system.

Another model of nonequilibrium thermometry that has been studied $[6,26,27]$ is pictorially displayed in Fig. 1. Consider a nonequilibrium system under thermal conduction because of the applied temperature difference $\Delta T=T^{H}-T^{C}$ 


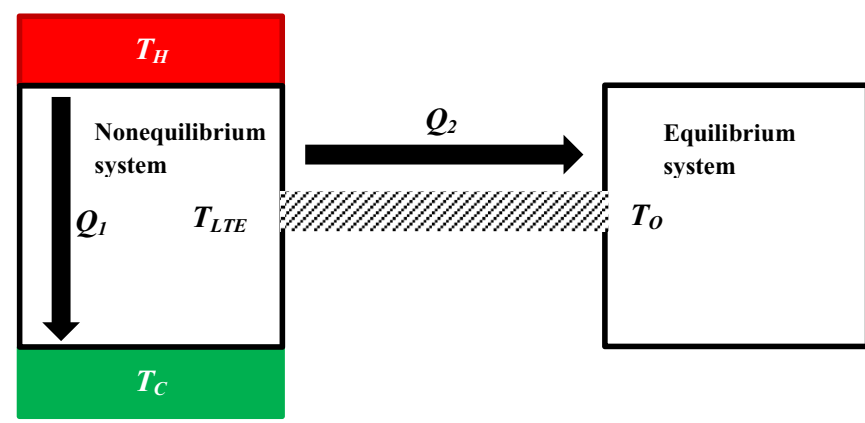

FIG. 1. The system on the left is in a nonequilibrium state whose temperature at a certain location we wish to measure. The system on the right is in equilibrium and behaves like a thermometer. A heat flux, $Q_{1}$, occurs in the nonequilibrium system because of the applied hot and cold temperatures, $T_{H}$ and $T_{C}$, respectively, at the top and the bottom ends. The shaded region represents a conductor that links the nonequilibrium system with the equilibrium system. If the temperatures at the two ends of the conductor, $T_{\mathrm{LTE}}$ and $T_{O}$, are not the same, then there is a heat flux $Q_{2}$. The operational temperature $[6,26,27]$ measured by the thermometer is defined as the temperature $T_{O}$ for which $Q_{2}=0$.

resulting in a finite heat flux $Q_{1}$. For simplicity, we assume that the system is in local thermodynamic equilibrium so that the local temperature of the system may be defined. Consider any location within the system having temperature $T_{\mathrm{LTE}}$. We now bring an equilibrium system, characterized by the temperature $T_{O}$, in contact with the nonequilibrium system by means of a conductor. The conductor, whose one end is at $T_{\mathrm{LTE}}$ and the other is at $T_{O}$, encounters a heat flux $Q_{2}$. The essence of the idea shown in Fig. 1 lies in tuning the equilibrium temperature $T_{O}$ such that $Q_{2}=0$. This concept of temperature measurement resembles the Zeroth Law based ideal-gas thermometry where the system and the thermometer are in equilibrium and the heat flux equals zero.

Hatano and Jou tested this idea using two single-harmonic oscillators [6] - a forced single-harmonic oscillator representing the nonequilibrium system, and a single-harmonic oscillator representing the system in equilibrium. The role of the intermediate conductor is played by the quadratic and bistable coupling potentials between the two systems. They concluded that $T_{O} \neq T_{C, \mathrm{LTE}}>T_{K, \mathrm{LTE}} \neq T_{O}$ with

$$
T_{\mathrm{LTE}}-T_{O} \approx Q_{2}^{2}
$$

The "thermometer" gives a different reading depending upon the coupling potential, highlighting the importance of the nature of the interaction between the system and the thermometer and the nonlocal characteristics of the measured temperature. A similar concept was used by Ritort-a two-state spin system being driven out of equilibrium by a sinusoidal force coupled with an equilibrium oscillator-to show that $T_{O}$ reaches the maximum value when there is resonance between the system and the thermometer [28].

While conceptually simple and appealing, the model can be improved upon. The description of the conductor linking the system and the thermometer alters $T_{\mathrm{LTE}}$. In the implementation by Jou et al. [3-6], the forced oscillator may bring the second oscillator out of equilibrium and for the forcing frequency different from the system frequency, $T_{C} \neq T_{K}$. Under such conditions, the basic tenets of the local thermodynamic equilibrium are violated as the existence of $T_{\mathrm{LTE}}$ implies $T_{C}=T_{K}$. There could be spurious effects arising due to the nonequilibrium temperature being controlled by the Langevin thermostat $[29,30]$. Moreover, the results may change depending upon the temperature control algorithm, as recent studies have indicated that the dynamical properties of a system depend upon the algorithm used [31,32].

The simplicity and the uniqueness of the method prompted another investigation along similar lines. Rather than using a forced oscillator, Hoover and Hoover [7] used a twodimensional heat conducting $\phi^{4}$ chain with one end particle thermostatted at $T^{H}$ and the other at $T^{C}$ by means of two Nosé-Hoover thermostats. The particles lying between the two thermostatted particles evolve under the Newtonian dynamics. The link between the hot and the cold thermostatted particle is provided by a single intermediate particle to which the remaining intermediate particles are connected, and it functions as the thermometer. Under this nonequilibrium setting, the kinetic and the configurational temperatures of the thermostatted particles were found to be different, again suggesting that the Zeroth Law of thermodynamics may not exist in such nonequilibrium cases. The configurational temperature of the hot (cold) particle was found to be consistently less (more) than its kinetic counterpart. Despite the near equivalence of the kinetic temperature with the local thermodynamic equilibrium temperature, the kinetic and the configurational temperatures measured by the thermometers were found to be different. We conjecture that this could be due to an algorithmic artefact [25] as the results may change if configurational thermostats were used instead of the Nosé-Hoover thermostat.

\section{THE PRESENT MODEL}

Our proposed model collates the ideas discussed in the previous section and brings computational thermometry closer to real-life thermometry. The system studied is similar to that employed by Baranyai $[17,18]$, and the method of measuring temperature is inspired by that depicted in Fig. 1. Rather than having a single thermometer measure the temperature of the entire nonequilibrium system, we employ multiple thermometers embedded in the system that measure the local temperature as shown in Fig. 2. In scenarios where the system and the thermometer particles do not collide but achieve equilibration by doing work, the merit of ideal-gas thermometry may be tested by using this model. As the thermometer particles constitute only a small fraction (6\%) of the system particles, this model of thermometry does not alter the system state once the steady state sets in, unlike in the previous studies. After initial transients have died out, the thermometers achieve steady-state conditions with a subregion of the system, and they enable one to find the temperature according to the different definitions. Further, unlike previous studies, the model does not treat any temperature definition preferentially.

The details of the simulation are as follows. Each particle is assigned a unit mass. We set $k_{B}=1$, employ nondimensional standard LJ units throughout and do not distinguish between the system and the thermometer particles at the beginning. 


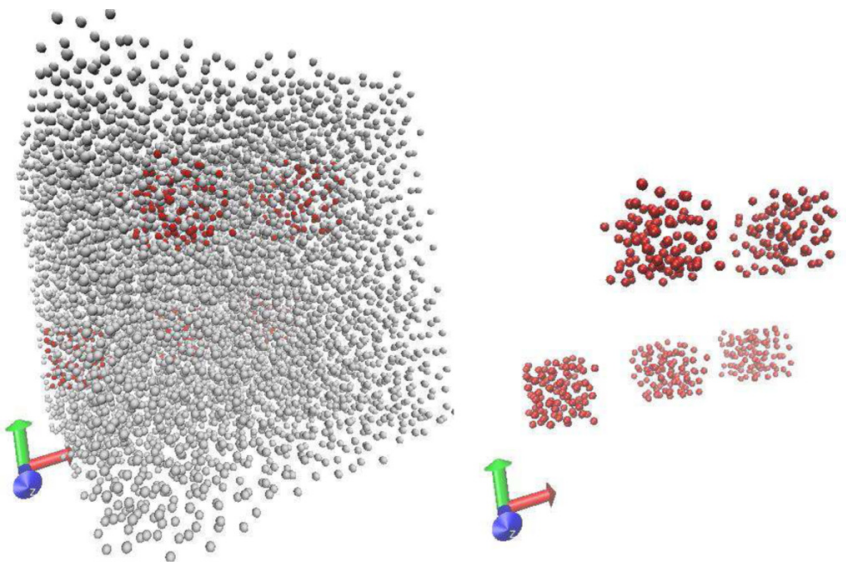

FIG. 2. The present model for measuring temperature in a nonequilibrium system. The model closely represents how we measure the temperature of a real system. The gray particles (on the left) are the system particles, while the red particles (on the right) represent the thermometers. The thermometer particles are embedded within the system and are tethered to their positions so that they can measure the local temperature. Overall, the system is divided into five equally spaced regions along the $x$ direction, and a thermometer is included in each region. The locations of the thermometers are staggered to avoid any interaction among them. For a cube of side length $L$, each thermometer has a dimension of $(L / 5, L / 5,3 L / 10)$. The approximate locations of the centroid of the thermometer particles are as follows: $(L / 10,3 L / 10, L / 4),(3 L / 10,7 L / 10,3 L / 4)$, $(L / 2,3 L / 10, L / 4),(7 L / 10,7 L / 10,3 L / 4)$, and $(9 L / 10,3 L, 10, L / 4)$.

The initial positions of all particles are randomly assigned in the simulation domain, which is a cube with side lengths determined by the desired number density, $\rho$. The nonperiodic reflecting boundary conditions along the three axes are enforced; if a particle crosses a simulation edge, the appropriate component of velocity is reversed, and the particle's position is updated within the simulation domain. All particles interact with each other through the LJ potential,

$$
U_{i j}=\left\{\begin{array}{cc}
4 \epsilon_{m n}\left[\left(\frac{1}{r_{i j}}\right)^{12}-\left(\frac{1}{r_{i j}}\right)^{6}\right], & r_{i j} \leqslant 2.5, \\
0, & r_{i j}>2.5,
\end{array}\right.
$$

where $r_{i j}$ equals the relative position of particle $j$ with respect to that of particle $i$. If the $i$ th particle corresponds to the system, then $m=0$, otherwise $m=1$. Likewise, $n$ equals 0 or 1 depending on if the $j$ th particle represents the system or the thermometer. We set $\epsilon_{11}=\epsilon_{00}=1$, and $\epsilon_{01}=\epsilon_{10}$ is assigned a value between 0 and 1 that reflects the interaction between the system and the thermometer particles.

Following a conjugate-gradient-based minimization and 50000 equilibration time steps, the system and the thermometer particles are tagged. In each region, $6 \%$ of the particles are demarcated as the thermometer particles. Once tagged, the system and the thermometer particles still interact according to the LJ potential, however the interaction between the system and the thermometer particles changes. The latter is also modeled through Eq. (10), but with varying values of $\epsilon_{01}=$ $\epsilon_{10}$-we investigate four cases with $\epsilon_{01}=0.25,0.50,0.75$, and 1.0 - to gather insights into the effect of interaction between the system and the thermometer particles. The simplicity of the LJ potential, along with its long-range attraction and short-ranged repulsion that mimics real-life systems, prompted us to use it for modeling purposes. We choose a cutoff distance of 2.5 where the truncation energy is $-0.016 \times \epsilon_{m n}$. Although a discontinuity exists at this point, for the present study it is expected not to significantly affect the results. Additionally, the thermometer particles are tethered to their initially tagged position by a harmonic potential:

$$
V\left(r_{i}\right)=\frac{1}{2}\left[r_{i}(t)-r_{i}\left(t_{e}\right)\right]^{2},
$$

where $r_{i}(t)$ denotes the instantaneous position of the $i$ th thermometer particle, and $r_{i}\left(t_{e}\right)$ denotes the initial tagged position of the same particle after the 50000 equilibration time steps. The tethering potential serves two purposes: (i) it holds the thermometer particles together tethered at one place, and (ii) it prevents a rigid body motion of the tethered particles. Thus our thermometer measures the local temperature of a region, unlike that of Baranyai's.

The simulation domain is divided into five regions of equal size along the $x$ axis, and each region has an embedded thermometer that measures the "local" temperature of the region. Thermometer particles are located in staggered positions in the five regions to minimize their influence on each other. The system particles of the leftmost and the rightmost regions are thermostatted at temperatures $T^{H}$ and $T^{C}$, respectively, which drives heat flow through the system. Thermostatting is done using two Nosé-Hoover thermostats [33]. It should be noted that rather than thermostatting the system particles themselves, we thermostat the regions so that as soon as a system particle leaves or enters the thermostatted region, it is either devoid or under the influence of the thermostat. The motion of the system particles in the three intermediate regions is governed by the Hamiltonian evolution equation. All thermometer particles are free from any thermostat influence.

The evolution equations of motion are integrated using the modified velocity-Verlet algorithm [34] for $1.5 \times 10^{6}$ time steps, with each step size $=0.001$. The thermostat masses for both the cold and the hot reservoirs are taken to be 500. For each region and thermometer, we separately compute $T_{K}, T_{C}$, $T_{R}$, and $T_{C, 2}$ at every time step. While evaluating $T_{C, 2}$, we have used the entire potential energy, $U$, of the system rather than the potential energy of a particular region. The results presented in this work are time averages of 1.45 million values. We have investigated the effects of (i) $\Delta T=T^{H}-T^{C}$; (ii) number density, $\rho$; (iii) interaction; and (iv) the system size, $N$, of the number of particles on the computed temperature values. The different configurations investigated are listed in Table I.

\section{RESULTS AND DISCUSSION}

\section{A. Temperature profile due to the NH thermostat}

Thermal conduction studied using the Nosé-Hoover thermostats has interesting temperature profiles $[7,25]$ and may be used to address the specific questions asked before. We illustrate these features through Fig. 3, which shows the temperature of the system particles present in the five regions for $\epsilon_{m \neq n}=1, \rho=0.728, N=5000$, and $\Delta T=2.0$. There is a $4 \%$ difference between the kinetic and the configurational temperatures of the hotter thermostatted region. All nonlocal measures of temperature are almost identical $-T_{C}, T_{R}$, and 
TABLE I. Details of the simulation study. Overall, 144 simulations were carried out to understand the effects of the system size, the density, the interaction, and the temperature difference $(\Delta T=$ $T^{H}-T^{C}$ ) on the temperature being measured by a thermometer. $N$ equals the total number of particles (system + thermometer).

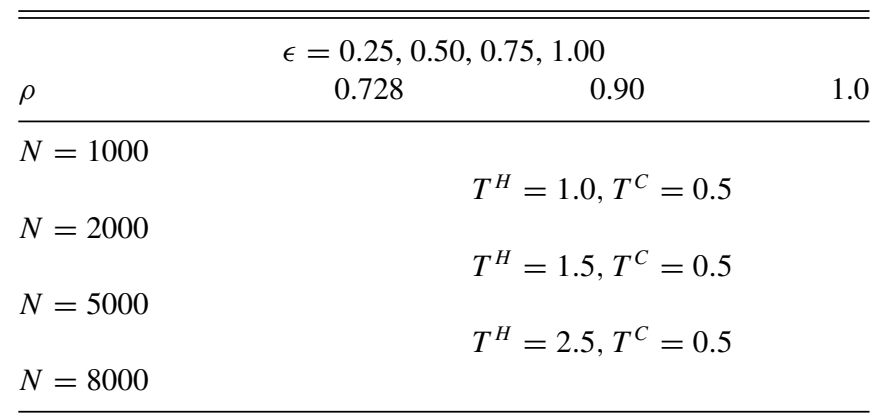

$T_{C, 2}$ do not have any appreciable differences among them. Thus, for the remainder of this paper, we report only the configurational temperature.

\section{B. Existence of LTE}

The nonequivalence of the kinetic and the configurational temperatures at the hot thermostatted end poses a question on whether the LTE conditions prevail within the system. We therefore check for the LTE conditions before proceeding further. If the system is in LTE, the momentum of the particles follows locally the Maxwell-Boltzmann distribution:

$$
f_{\mathrm{LTE}}\left(p_{1}, p_{2}, \ldots, p_{3 N^{\prime}}\right) \propto \exp \left[\frac{1}{2 T_{\mathrm{LTE}}} \sum_{i=1}^{3 N^{\prime}} p_{i}^{2}\right]
$$

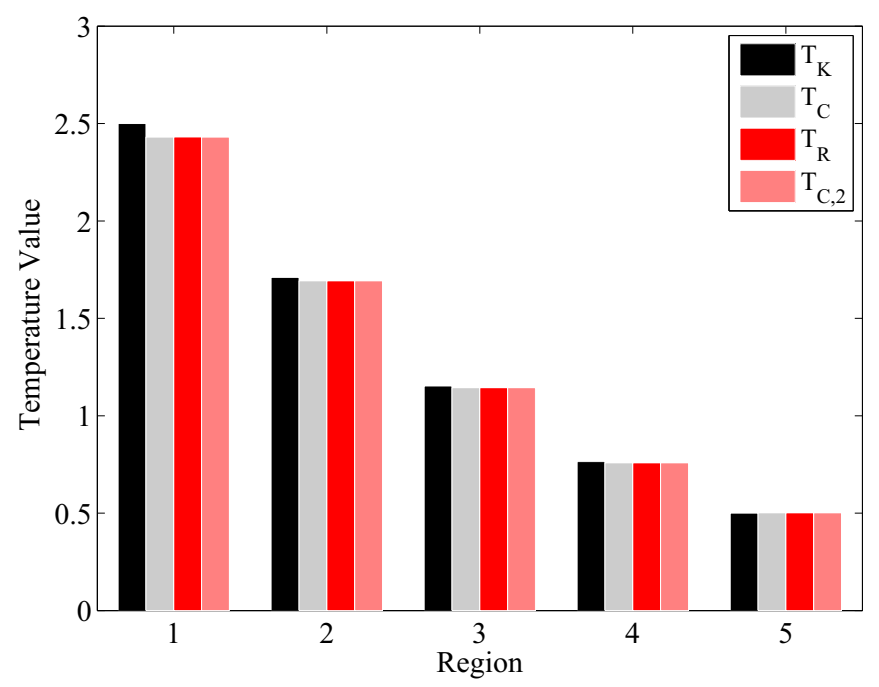

FIG. 3. The temperatures of the five regions with $\epsilon_{m \neq n}=1, \rho=$ $0.728, N=5000$, and $\Delta T=2.0$. Note that these results are only for the system particles. There is a substantial difference between the kinetic and the configurational temperatures at the hot thermostatted region. A similar observation was found for all other systems as well. As one approaches the colder region, the difference between the kinetic and the configurational temperatures decreases.

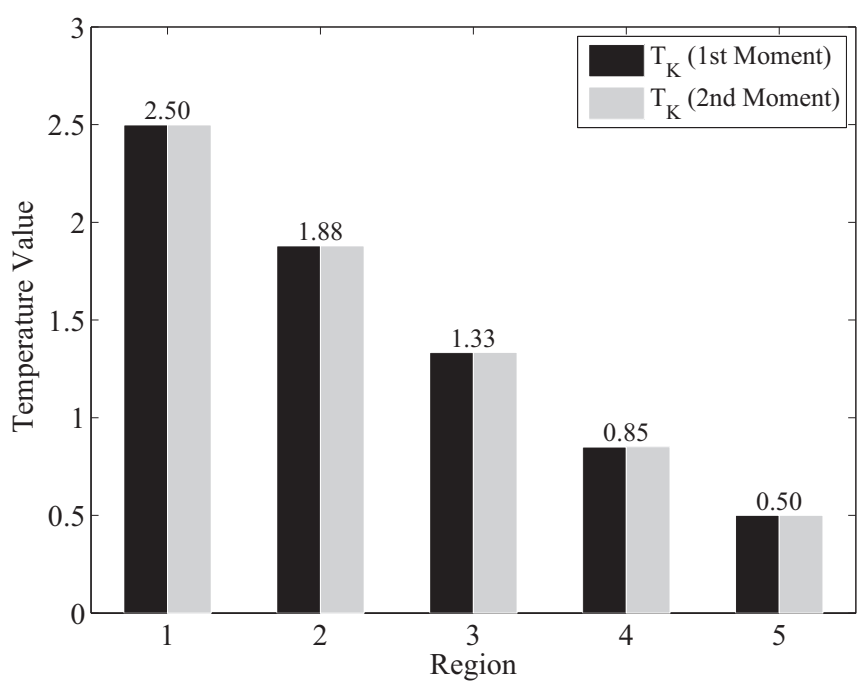

FIG. 4. The near equivalence of $T_{\mathrm{LTE}}$ from the first (black bars) and the second (gray bars) moments of kinetic energy. The system under investigation corresponds to $\epsilon_{m \neq n}=1, \rho=0.728, N=5000$, and $\Delta T=2.0$. This figure shows that locally the momentum distribution of the particles follows the Maxwell-Boltzmann distribution, and hence the system is in local thermodynamic equilibrium. Local thermodynamic equilibrium conditions prevail despite the small but substantial difference between the kinetic and the configurational temperatures, as seen in Fig. 3.

In other words, the linear momentum $p_{i}=m v_{i}$ of the particles in a particular region has a normal distribution with a variance equal to $T_{\mathrm{LTE}}$. Consider a random variable $Z_{i}=p_{i} / \sqrt{T_{\mathrm{LTE}}}$. It is evident that $Z_{i}$ follows a standard normal distribution. Thus, $\sum_{i=1}^{3 N^{\prime}} Z_{i}^{2}$ follows a $\chi^{2}$ distribution with $3 N^{\prime}$ degrees of freedom. To see if the system is in local thermodynamic equilibrium, we check for the near equivalence of the $T_{\mathrm{LTE}}$ as calculated from the first two central moments of the resulting $\chi^{2}$ distribution. The equations to be solved are as follows:

$$
\begin{gathered}
\text { First moment } \rightarrow\langle Y\rangle=3 N^{\prime} T_{\mathrm{LTE}}, \\
\text { Second moment } \rightarrow\left\langle Y^{2}\right\rangle-\langle Y\rangle^{2}=6 N^{\prime} T_{\mathrm{LTE}}^{2},
\end{gathered}
$$

with $Y=\sum_{i=1}^{3 N^{\prime}} p_{i}^{2}$.

The LTE conditions prevailing within the system can be gauged from the near-equivalence of the $T_{\text {LTE }}$ shown in Fig. 4. A fundamental consequence of the existence of the LTE is the applicability of postulates of equilibrium thermodynamics locally, including the equality of the different temperature measures. However, as is evident from Fig. 3, in our case a situation arises where at the boundaries (i.e., the thermostatted regions), the postulates of equilibrium thermodynamics are not applicable despite the existence of the LTE, while away from the boundaries the results are as expected. This simultaneous violation and agreement with the tenets of the LTE hypothesis enable us to judge the merits of the nonequilibrium thermometry without looking at far-from-equilibrium situations by checking whether the thermometers "sense" the kinetic temperature or the configurational temperature of the system. An agreement between the different temperature definitions 


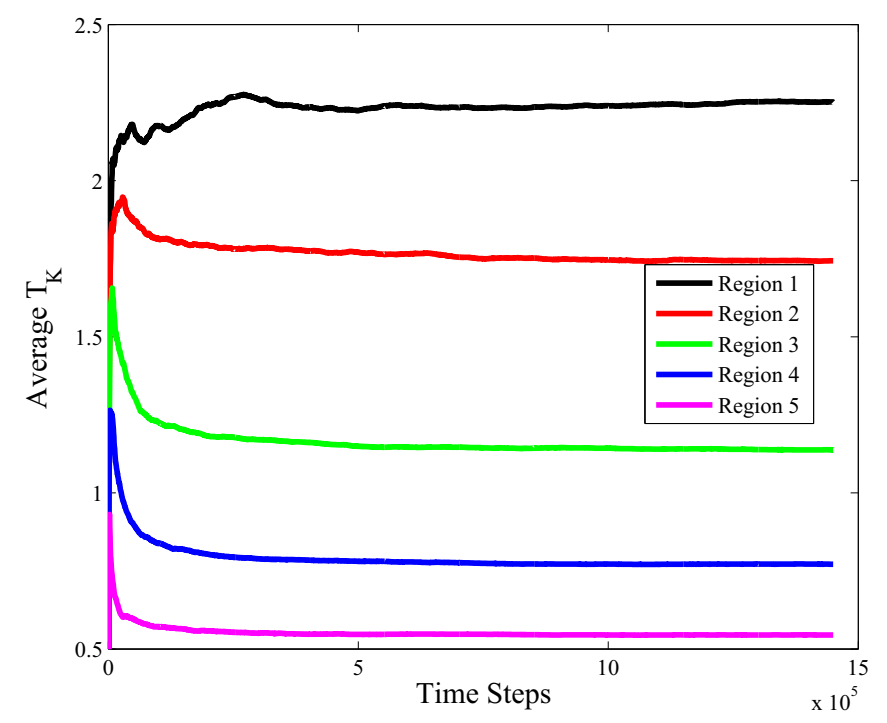

FIG. 5. For $\epsilon_{m \neq n}=1, \rho=0.728, N=5000$, and $\Delta T=2.0$, the temporal evolution of the average kinetic temperature measured by the thermometers embedded in the five regions. For each region, it is evident that the thermometer has reached a steady state.

of the system and the thermometer indicates the compliance of the Zeroth Law in the LTE situations. A disagreement, on the other hand, suggests the violation of the Zeroth Law. When immersed in the system, the energy is transferred to the thermometers through their interaction with the system particles. The interaction is nonlocal, i.e., the thermometer particles feel effects of all the system particles lying within the cutoff range. Consequently, it is not easy to predict what temperature a thermometer would measure, especially when the kinetic and the configurational temperatures are different in the thermostatted regions.

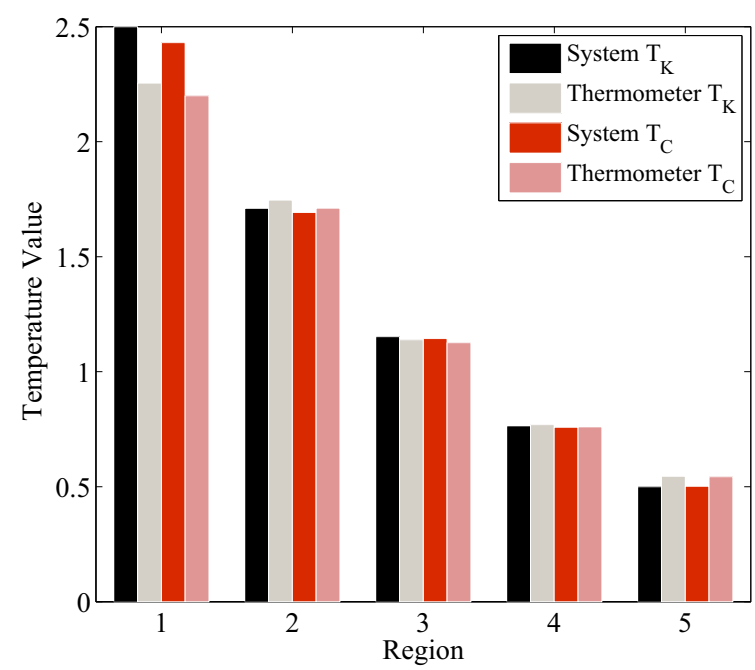

\section{Steady states exist within the thermometers}

In real-life thermometry, after initial transients, a thermometer attains equilibrium with the system and the corresponding reading is ascribed the name "temperature." The reading does not show any appreciable fluctuations with time. In the present model of nonequilibrium thermometry a similar situation occurs, as can be seen from Fig. 5, in which we have plotted the temporal evolution of the average kinetic temperature for the case $\epsilon_{m \neq n}=1, \rho=0.728, N=5000$, and $\Delta T=$ 2.0. Upon coming in contact with the system, each locally embedded thermometer shows transient thermal conduction, and after $1.5 \times 10^{6}$ time steps it shows steady-state conditions where the temperature does not significantly fluctuate. The rate of approach to steady-state conditions depends on the region where the thermometer is present-whereas for regions 4 and 5 the temperature reaches a steady-state value after $0.5 \times 10^{6}$ time steps, it takes nearly $1.5 \times 10^{6}$ time steps for the other regions.

\section{Temperature measured by the thermometers}

Figure 6 shows the temperatures measured by the thermometers as well as their difference from the LTE temperature ( $\left.T_{\mathrm{LTE}}\right)$ corresponding to $\epsilon_{m \neq n}=1, \rho=0.728, N=5000$, and $\Delta T=2.0$. Interestingly, at the thermostatted ends, there is a substantial difference (around 10\%) between $T_{\text {LTE }}$ and both the kinetic and the configurational temperatures of the thermometer $\left(\Delta T_{K}\right.$ and $\Delta T_{C}$, respectively). The thermometers in these regions measure neither $T_{K}$ nor $T_{C}$. The nonequivalence of the temperatures with $T_{\mathrm{LTE}}$ questions the existence of the Zeroth Law in these regions. These results agree with those of Jou and Hatano [6], where $T_{K}$ and $T_{C}$ of the oscillators are not the same.

However, as we move inward from the ends, we see that there is a negligible difference $(\leqslant 2 \%)$ between $T_{K}, T_{C}$, and $T_{\mathrm{LTE}}$, and the thermometer correctly identifies $T_{\mathrm{LTE}}$. The

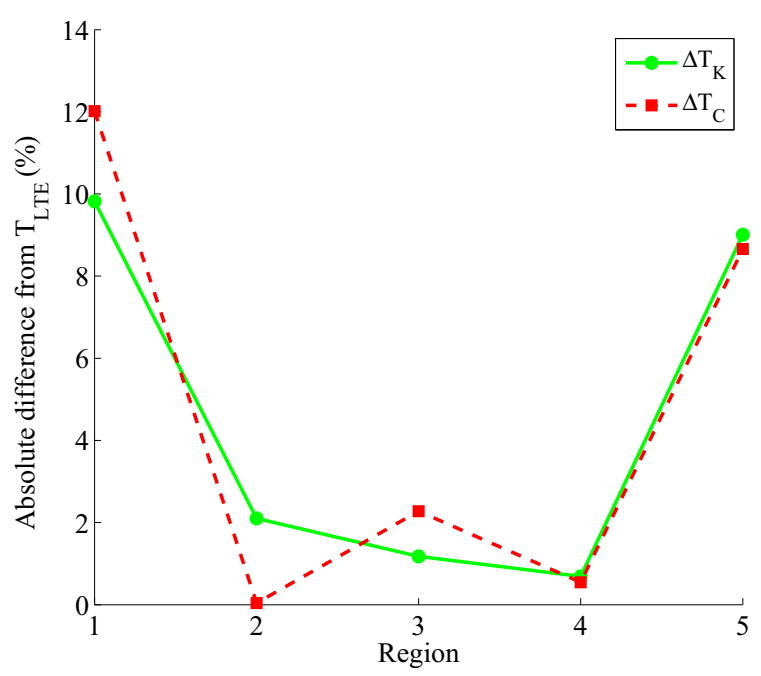

FIG. 6. Left: Comparison of the kinetic (black bars) and the configurational (red bars) temperatures measured by the thermometers with the kinetic (gray bars) and the configurational (light red bars) temperatures of the system. This case corresponds to $\epsilon_{m \neq n}=1, \rho=0.728$, $N=5000$, and $\Delta T=2.0$. Right: The difference of the kinetic and the configurational temperatures from the local thermodynamic equilibrium temperatures $\left(\Delta T_{K}\right.$ and $\Delta T_{C}$, respectively) in the five regions. 


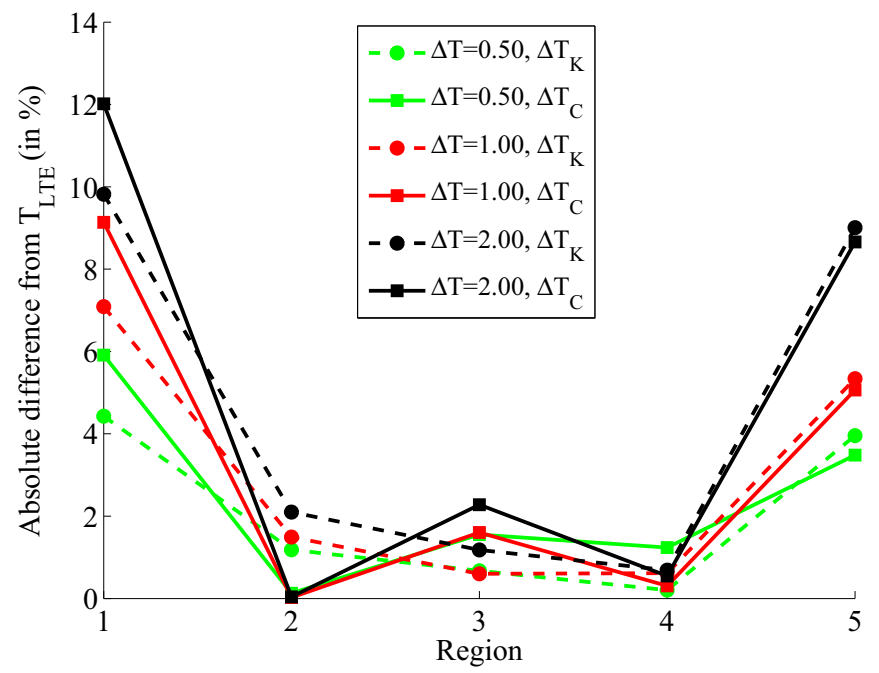

FIG. 7. Absolute difference of the kinetic and the configurational temperatures measured by the thermometer from the LTE temperatures. This case corresponds to $\epsilon_{m \neq n}=1, \rho=0.728$, and $N=5000$. Like the results depicted in Fig. 6, we observe that there is a very small difference between the kinetic (and the configurational) temperatures and the LTE temperatures in the inner regions. The thermometers accurately measure the LTE temperature of the system only in the inner regions. However, at the thermostatted ends, the deviations from the $T_{\mathrm{LTE}}$ increase with an increase in $\Delta T$.

difference between $T_{K}$ and $T_{C}$ of the thermometers in regions 2,3 , and 4 is small enough to conclude that within the thermometer, the Zeroth Law is satisfied among the kinetic and the configurational degrees of freedom. Further, the Zeroth Law holds between the system and the thermometer as well.

A similar conclusion could be drawn for the other values of $\Delta T$, as can be seen from Fig. 7 , which shows the deviations of the $T_{K}$ and the $T_{C}$ of the thermometers from the $T_{\mathrm{LTE}}$. As in Fig. 6, we observe that the thermometer accurately measures $T_{\mathrm{LTE}}$ for the inner regions. In fact, the deviation of $T_{K}$ and $T_{C}$ from $T_{\text {LTE }}$ is negligible $(\leqslant 2 \%)$.

Figure 8 shows the difference in the temperatures measured by the thermometers and the $T_{\mathrm{LTE}}$ with an increase in the density of the system. The system size is kept fixed at 5000 particles and $\Delta T=2.0$. While in the inner regions the thermometer accurately captures the LTE temperature, in the thermostatted outer regions, with an increase in $\rho$, the deviations of the $T_{K}$ and the $T_{C}$ from the $T_{\mathrm{LTE}}$ shows no improvement. Interestingly, $T_{C}$ and $T_{K}$ measured by the thermometers in the thermostatted regions are almost the same at higher densities.

It has been argued before that the interaction between the thermometer and the system influences the sensing capabilities of the thermometer [6]. We now investigate this feature by changing the interaction between the system particles and the thermometer particles. We set $\epsilon_{m \neq n}=0.25,0.5,0.75$, and 1.0 for this purpose. Figure 9 shows the effect of changing the interaction while keeping all other variables constant. It is interesting to note that with increasing interaction between the system and the thermometer particles, the ability of the thermometer to sense the LTE temperature improves, and

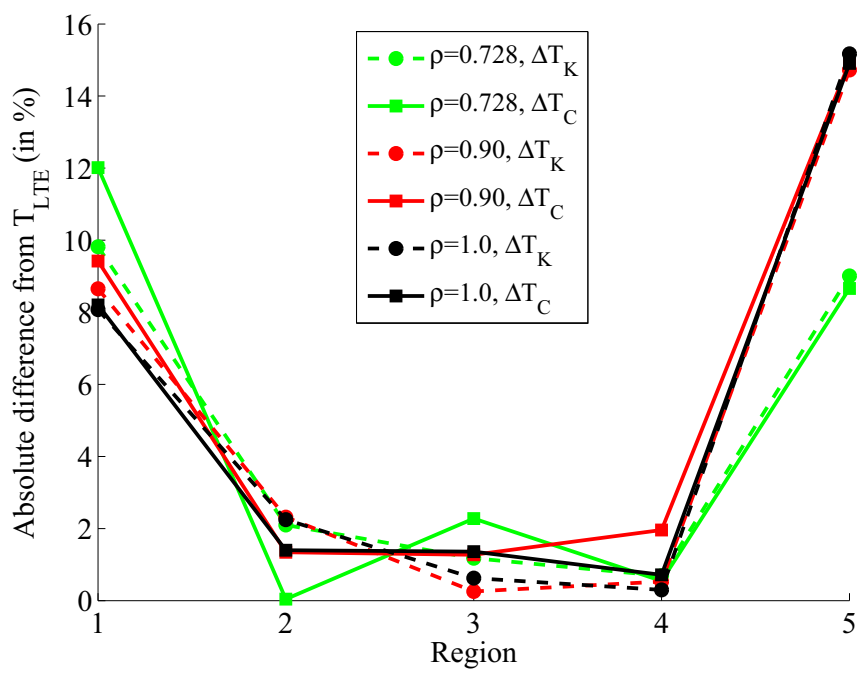

FIG. 8. For $\epsilon_{m \neq n}=1, \Delta T=2.0$, and $N=5000$, the absolute deviation of the kinetic and the configurational temperatures measured by the thermometer from the LTE temperatures. In the thermostatted regions, the difference from the $T_{\mathrm{LTE}}$ is higher than that observed in Fig. 7, but in the inner regions we see that the thermometers accurately measure $T_{\mathrm{LTE}}$.

away from thermostatted ends the deviation in certain cases is smaller than $2 \%$.

To ensure that the system with $N=5000$ gives reasonably accurate results, we study the effect of the system size, keeping other variables constant on the thermometer readings. The results are depicted in Fig. 10. Although we do not observe any particular trend in the deviation with changing system size in the thermostatted regions, Fig. 10 shows that the results with $N=5000$ and 8000 are qualitatively and quantitatively close to each other. Thus, the results presented above with

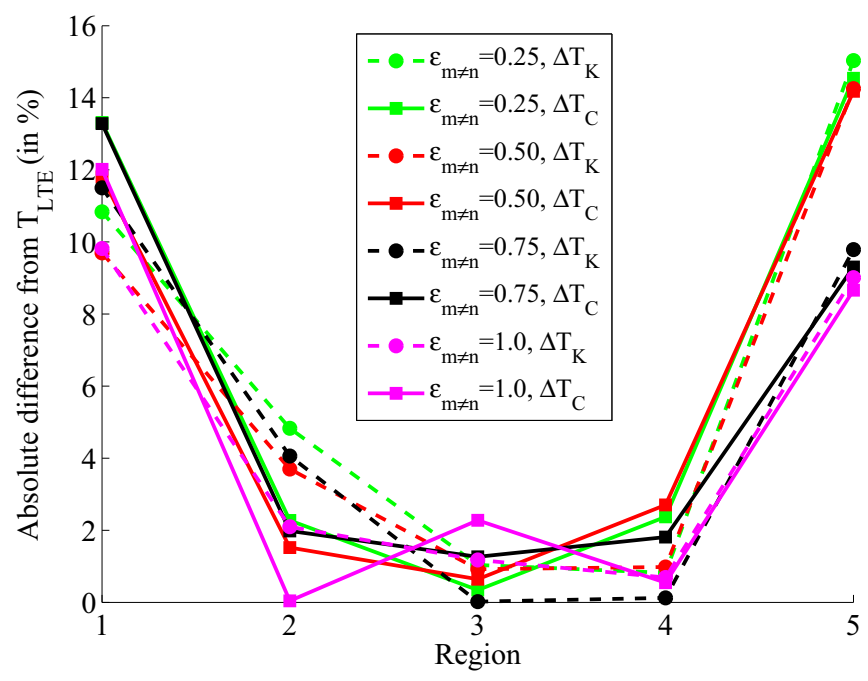

FIG. 9. For $T^{H}-T^{C}=2.0, \rho=0.728$, and $N=5000$, the absolute deviation of the kinetic and the configurational temperatures measured by the thermometer from the LTE temperature. In the thermostatted regions, the deviations of the $T_{K}$ and the $T_{C}$ increase with an increase in $\rho$ but remain unaltered in the inner regions. That is, in the inner regions, the thermometers accurately measure $T_{\mathrm{LTE}}$. 


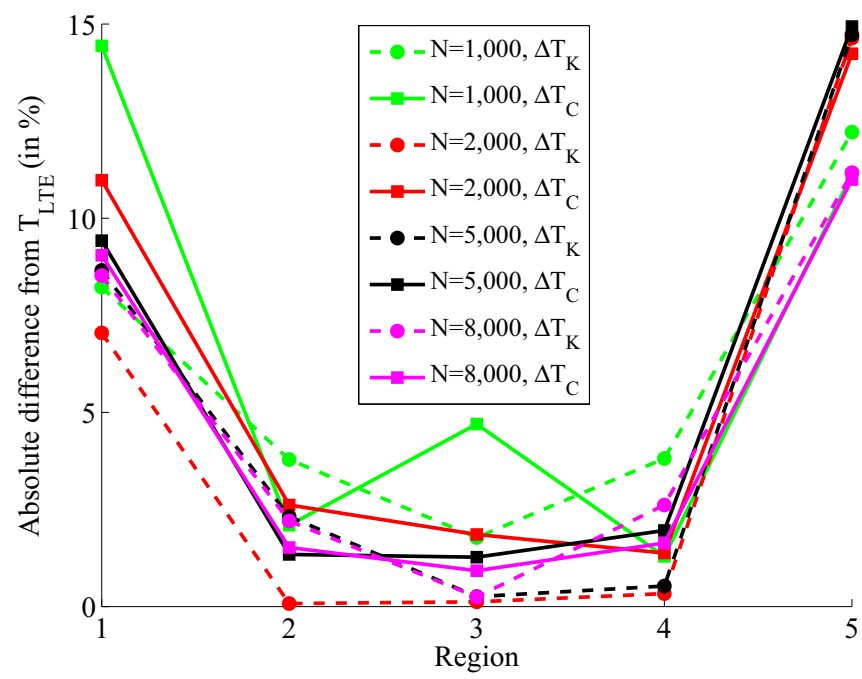

FIG. 10. Deviations of $T_{K}$ and $T_{C}$ of the thermometers from the $T_{\text {LTE }}$ with changing $N$, keeping other variables constant: $\rho=0.90$, $\Delta T=2.0$, and $\epsilon_{m \neq}=1.0$. We do not observe any particular trend in the deviation with changing system size.

$N=5000$ are representative of those for a larger number of particles.

\section{CONCLUDING REMARKS}

Ideal-gas thermometry is based on the equilibration between the system and the thermometer particles achieved through elastic collisions. It thus predicts that the temperature measured by a thermometer equals the average kinetic energy of the system. However, in realistic scenarios, two particles need not collide with each other. In such cases, the fundamentals of ideal-gas thermometry are not clear. It still works extremely well for equilibrium cases because of the equality of the different measures of temperature. But, in nonequilibrium regimes where defining a unique temperature is problematic, along with the fact that particles may not collide with each other, it is not clear what temperature a thermometer measures. In this work, we have tested the merits of ideal-gas thermometry by embedding multiple thermometers in a heat conducting system. These thermometers behave like real-life thermometers-(i) they are small enough (they comprise only $6 \%$ of the system particles) not to alter the steady state of the nonequilibrium system, (ii) any two particles never collide, and (iii) after showing initial transients, they provide steady-state values of the different measures of temperature. The nonequilibrium problem studied is a thermal conduction process affected by two Nosé-Hoover $(\mathrm{NH})$ thermostats. The interesting temperature profiles due to the $\mathrm{NH}$-based thermal conduction enable us to explore both cases $T_{K} \neq T_{C}$ and
$T_{K} \approx T_{C}$ locally, without looking at far-from-equilibrium dynamics. Further, we show that local thermodynamic equilibrium conditions prevail in these systems, which makes possible the definition of a unique local thermodynamic equilibrium temperature, $T_{\mathrm{LTE}}$.

The key takeaways from this investigation are as follows: (i) the Zeroth Law is satisfied in the regions where $T_{C} \approx T_{K}$ locally, whereas for the regions where $T_{C} \neq T_{K}$ the Zeroth Law does not hold despite the existence of the $T_{\mathrm{LTE}}$; (ii) the validity of the Zeroth Law is crucial for the thermometers to sense the correct system temperature, as for the regions where $T_{K} \neq T_{C}$ the thermometers do not correctly identify either $T_{C}$ or $T_{K}$; (iii) at the thermostatted ends, $T_{C} \neq T_{K}$ for the thermometers despite them being free from the influence of the thermostats; (iv) the temperature measured by a local thermometer depends marginally on the interaction between the system and the thermometer; and (v) a unique temperature measurement by a thermometer under nonequilibrium settings depends upon $T_{K}$ being nearly equal to $T_{C}$. Studies based on fewer degrees of freedom may have come to a different conclusion because of the effects of the finite size. In our study as well, for cases with a small number of particles, the configurational temperature measured by the thermometer is different from the LTE temperature. However, the difference decreases with an increase in the system size. Thus, if one extrapolates these results to the thermodynamic limit, one should not encounter any major difficulty in nonequilibrium thermometry. It is noteworthy that regardless of the configurational temperature of the system, the thermometer has a configurational temperature commensurate with its kinetic counterpart at larger system size even at thermostatted ends.

Further investigation is necessary to state if the inequality between the measured temperature and the local thermodynamic equilibrium temperature at the thermostatted ends is a general phenomenon. One may analyze such a situation by creating a case in which the LTE is deliberately violated and coupling it with the present thermometer model. An example of the LTE violating system is the differential thermal conduction model [35] where the kinetic and the configurational temperatures of a thermostatted end are kept at different values. We conjecture that our results may be useful for researchers studying other nonequilibrium processes such as Couette flow. However, a separate study needs to be conducted to confirm this.

\section{ACKNOWLEDGMENTS}

The authors are indebted to William Graham Hoover and the two anonymous reviewers for providing constructive comments on an earlier version of the manuscript that have helped improve upon the presentation of the work.
[1] S. R. De Groot and P. Mazur, Non-equilibrium Thermodynamics (North-Holland Pub. Co, New York, 1962).

[2] I. Gyarmati, Non-equilibrium Thermodynamics: Field Theory and Variational Principles (Springer, Berlin, 1970).
[3] J. Casas-Vázquez and D. Jou, Phys. Rev. E 49, 1040 (1994).

[4] D. Jou, J. Casas-Vázquez, and G. Lebon, in Extended Irreversible Thermodynamics (Springer, Berlin, 1996), pp. 41-74.

[5] J. Casas-Vázquez and D. Jou, Rep. Prog. Phys. 66, 1937 (2003). 
[6] T. Hatano and D. Jou, Phys. Rev. E 67, 026121 (2003).

[7] W. G. Hoover and C. G. Hoover, Phys. Rev. E 77, 041104 (2008).

[8] G. Lebon, D. Jou, and J. Casas-Vázquez, Understanding Nonequilibrium Thermodynamics (Springer, Berlin, 2008).

[9] B. Hafskjold and S. K. Ratkje, J. Stat. Phys. 78, 463 (1995).

[10] J. Xu, S. Kjelstrup, and D. Bedeaux, Phys. Chem. Chem. Phys. 8, 2017 (2006).

[11] J. Powles, G. Rickayzen, and D. Heyes, Mol. Phys. 103, 1361 (2005).

[12] B. L. Holian, W. G. Hoover, and H. A. Posch, Phys. Rev. Lett. 59, 10 (1987).

[13] W. G. Hoover and C. G. Hoover, Time Reversibility, Computer Simulation, Algorithms, Chaos (World Scientific, Singapore, 2012), Vol. 13 .

[14] P. K. Patra and B. Bhattacharya, J. Chem. Phys. 142, 194103 (2015).

[15] B. L. Holian, W. G. Hoover, B. Moran, and G. K. Straub, Phys. Rev. A 22, 2798 (1980).

[16] W. G. Hoover, B. L. Holian, and H. A. Posch, Phys. Rev. E 48, 3196 (1993).

[17] A. Baranyai, Phys. Rev. E 61, R3306 (2000).

[18] A. Baranyai, Phys. Rev. E 62, 5989 (2000).
[19] B. Butler, G. Ayton, O. G. Jepps, and D. J. Evans, J. Chem. Phys. 109, 6519 (1998).

[20] C. Braga and K. P. Travis, J. Chem. Phys. 123, 134101 (2005).

[21] D. J. Evans and G. P. Morriss, Phys. Rev. Lett. 56, 2172 (1986).

[22] K. P. Travis, P. J. Daivis, and D. J. Evans, J. Chem. Phys. 103, 10638 (1995).

[23] O. G. Jepps, G. Ayton, and D. J. Evans, Phys. Rev. E 62, 4757 (2000).

[24] H. H. Rugh, Phys. Rev. Lett. 78, 772 (1997).

[25] P. Patra and B. Bhattacharya, J. Chem. Phys. 140, 064106 (2014).

[26] D. Jou and J. Casas-Vázquez, Phys. Rev. A 45, 8371 (1992).

[27] D. Jou and J. Casas-Vázquez, Phys. Rev. E 48, 3201 (1993).

[28] F. Ritort, J. Phys. Chem. B 109, 6787 (2005).

[29] A. Jones and B. Leimkuhler, J. Chem. Phys. 135, 084125 (2011).

[30] P. K. Patra, M. Meléndez, and B. Bhattacharya, Phys. Rev. E 92, 023304 (2015).

[31] J. E. Basconi and M. R. Shirts, J. Chem. Theor. Comput. 9, 2887 (2013).

[32] P. J. Daivis, B. A. Dalton, and T. Morishita, Phys. Rev. E 86, 056707 (2012).

[33] W. G. Hoover, Phys. Rev. A 31, 1695 (1985).

[34] S. Toxvaerd, Mol. Phys. 72, 159 (1991).

[35] P. K. Patra and B. Bhattacharya, Phys. Rev. E 93, 033308 (2016). 